\title{
Pancreatitis kills cysts: A phenomenon that illustrates the potential role of immune activation in premalignant cyst ablation
}

(이요 $\ominus$

\author{
Authors \\ Leonard T. Walsh ${ }^{1}$, Matthew T. Moyer ${ }^{2}$ \\ Institutions \\ 1 Penn State Health Milton S. Hershey Medical Center - \\ Internal Medicine, Hershey, Pennsylvania, United States \\ 2 Penn State Health Milton S. Hershey Medical Center - \\ Gastroenterology and Hepatology, Hershey, \\ Pennsylvania, United States
}

submitted 19.9.2019

accepted after revision 18.5 .2020

\author{
Bibliography \\ DOI https://doi.org/10.1055/a-1196-1343 | \\ Endoscopy International Open 2020; 08: E1039-E1041 \\ (c) Georg Thieme Verlag KG Stuttgart · New York \\ eISSN 2196-9736
}

Corresponding author

Leonard T. Walsh, MD, Penn State Health Milton S Hershey Medical Center - Internal Medicine, 500 University Drive Hershey Pennsylvania 17033-0850, United States
Fax: +1-717-531-2034
leonard.walsh@guthrie.org

\section{ABSTRACT}

Background and study aims Pancreatic cystic lesions are being increasingly recognized on cross-sectional imaging and mucinous premalignant cysts represent one of the most prevalent types. Endoscopic ultrasound (EUS)-guided chemoablation offers a safe and minimally invasive ablation approach yet with limited efficacy. Patients who develop acute pancreatitis as a complication of chemoablation with alcohol however experience ablation rates up to $100 \%$. This evidence suggests that recruitment of the body's immune system may represent an avenue to significantly increase the efficacy of EUS-guided chemoablation. Here we illustrate this immune-mediated tumor killing phenomenon through a case presentation at our institution.

\section{Introduction}

Pancreatic cystic lesions (PCLs) are being increasingly recognized on imaging studies, with prevalence increasing with age and ranging from $2 \%$ to $37 \%$ [1]. Mucinous cysts, including mucinous cystic neoplasms (MCNs) and intraductal papillary neoplasms (IPMNs), are the most prevalent type of pancreatic cysts and are at risk of progressing into invasive carcinoma. These cysts require further management, yet current strategies including radiographic surveillance and surgical resection present their own set of difficulties. Radiographic surveillance is associated with high cost, radiation exposure, and offers no definitive treatment, and surgical resection carries substantial risk for serious adverse events (AEs) (20\%-40\%) and mortality (1\%$3 \%$ ) [1]. Endoscopic ultrasound (EUS)-guided fine needle infusion is an evolving and promising method for chemically ablating appropriately selected mucinous type pancreatic cysts with an endoscopic, minimally invasive technique that carries complete ablation rates ranging from $50 \%$ to $79 \%$ [2-4]. Rates of associated serious AEs for EUS-guided chemoablation range from $0 \%$ using an alcohol-free technique to as high as $10 \%$ when alcohol is used in the ablation process [1-4]. The most common AE is pancreatitis, which is believed to be due to the inflammatory and toxic effects of the dehydrated alcohol [1].

Interestingly, although pancreatitis is considered an $\mathrm{AE}$, we note that in randomized trials where the treatment results of patients who suffered acute pancreatitis (AP) were reported, the cyst ablation rates of patients with AP were as high as $100 \%[1,5]$. There was complete ablation in the two trials in which these metrics were reported, yet when reexamining another randomized trial with the investigator, this phenomenon may not have been observed [6]. This evidence, however, suggests that localized recruitment of the immune system may represent an alternative treatment approach - the addition an immune stimulating agent to the chemoablation cocktail which could stand to significantly increase the efficacy of EUSguided chemoablation. Here we present a case at our institution that impressively illustrates the pancreatitis kills cysts phenomena. 


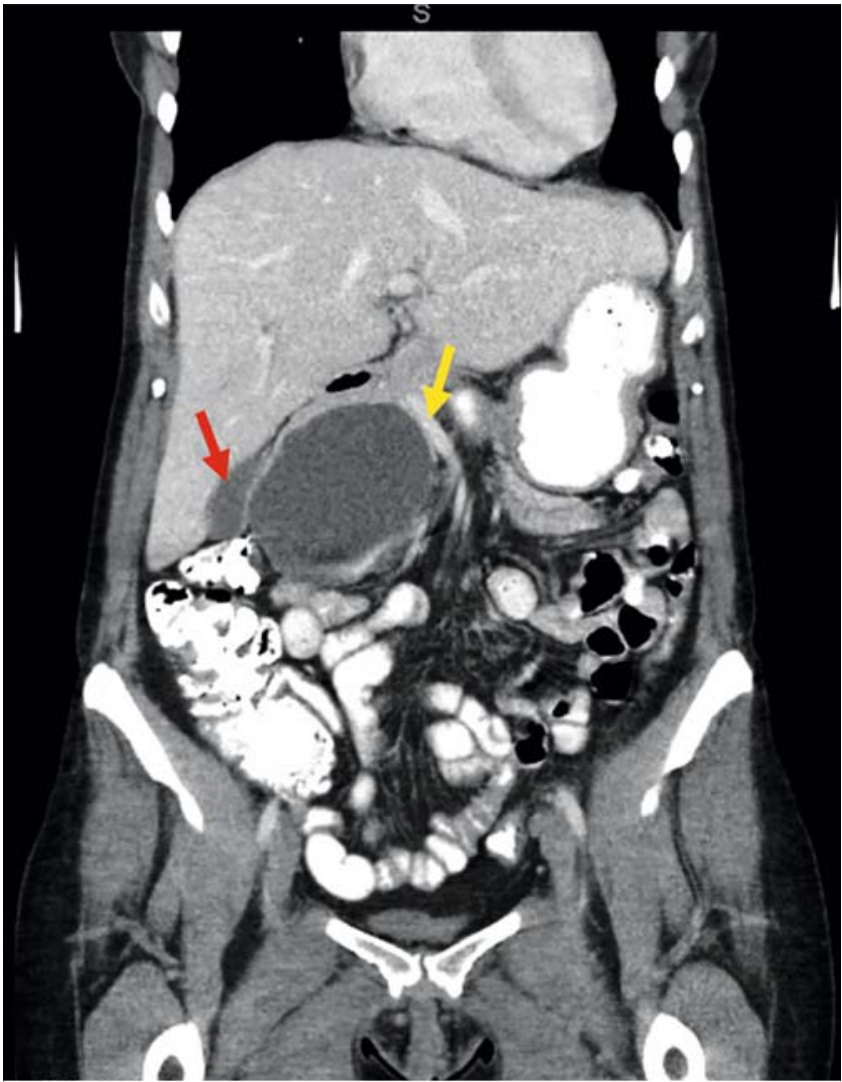

- Fig. 1 Coronal abdominal CT showing a $7.7 \mathrm{~cm} \times 6.7-\mathrm{cm}$ cystic pancreatic head lesion displacing the gallbladder (red arrow) portal and superior mesenteric veins (yellow arrow).

\section{Case report}

A 53-year-old female with no significant medical history noticed a palpable abdominal mass, and subsequent abdominal computed tomography $(\mathrm{CT})$ and magnetic resonance imaging (MRI) showed an approximate $8 \times 7 \mathrm{~cm}$ cystic mass ( $\bullet$ Fig. 1 and $>$ Fig. 2 ) in the head of the pancreas with internal septations and thin calcifications in the cyst wall. Fluid cytology showed pigment-laden macrophages and glandular debris. Carcinoembryonic antigen could not be obtained due to high viscosity of the cyst fluid. The cyst was most consistent with a high-risk MCN given the presentation in a middle-aged female with no prior history of pancreatitis and characteristic crosssectional imaging with lack of a competing diagnosis.

The patient had undergone a technically challenging EUSguided fine-needle aspiration (FNA) of the cyst at an outside institution, leading to multiple FNA needle exchanges. She subsequently developed acute pancreatitis leading to a 7-day hospitalization. After being offered a Whipple procedure (panceaticoduodenectomy), she presented to our institution for consideration of alcohol-free, EUS-guided chemoablation of her pancreatic cyst. However, magnetic resonance imaging (MRI) and magnetic resonance cholangiopancreatography (MRCP) 5 weeks after her hospitalization for pancreatitis showed a significantly smaller cyst of $3 \mathrm{~cm}$ without fistulization, fluid collections, or development of walled-off pancreatic necrosis

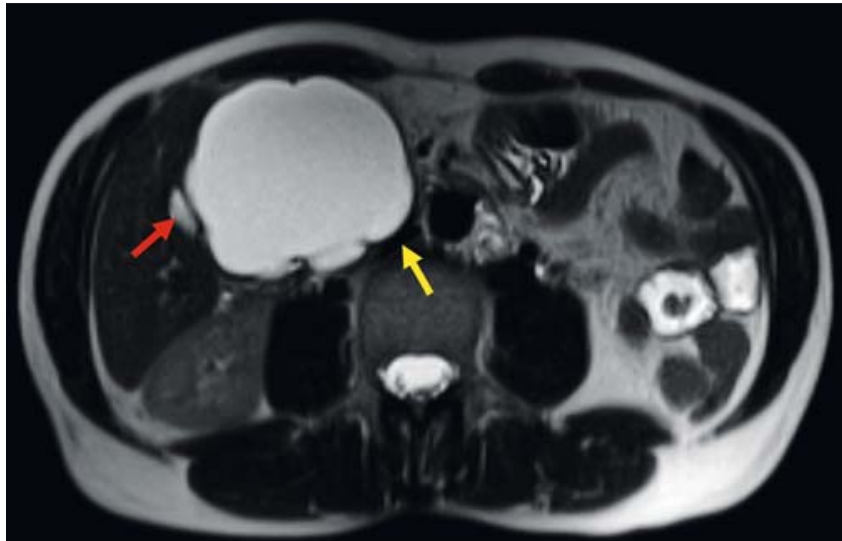

Fig. 2 T2-weighted MRI showing a $7.2 \mathrm{~cm} \times 6.6$ - $\mathrm{cm}$ cystic mass lesion of the pancreatic head with mass effect displacing the gallbladder (red arrow) and portal venous system (yellow arrow).

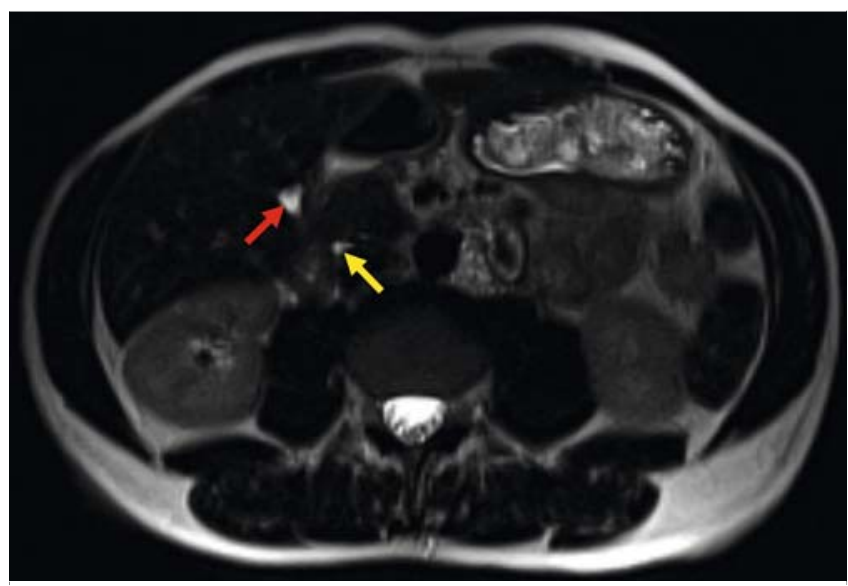

- Fig. 3 T2-weighted MRI at 18 months post FNA-induced pancreatitis showing complete resolution of the MCN. Cystic duct is shown by the red arrow and a non-dilated section of the pancreatic duct is shown by the yellow arrow.

(WOPN), and when the EUS-guided ablation procedure was performed at 11 weeks, no cyst could be found. Follow-up MRI-MRCP at 9 and 18 months ( $\triangleright$ Fig. 3 ) showed complete cyst resolution without development of further complications or other intraabdominal findings.

\section{Discussion}

Cyst ablation rates in patients who develop acute pancreatitis as a result of cyst chemoablation are reported near $100 \%$ in the literature versus $50 \%$ to $79 \%$ with EUS-chemoablation without development of pancreatitis. Here we illustrate the inflammatory phenomena of acute pancreatitis that leads to higher rates of cyst ablation, and speculate on the potential role of an immune-stimulating agent as an additive to the chemoablation cocktail which may stand to improve the efficacy of premalignant pancreatic cyst chemoablation.

Acute pancreatitis involves a complex cascade of immunologic events with recruitment of leukocytes including macropha- 
ges, monocytes, and T cells, and as such, the addition of an immune-activating agent to the chemoablation admixture by EUS-guided infusion may carry independent efficacy and have a synergistic effect on ablation rates.

Immunotherapy represents a recent paradigm shift in the treatment of malignancies and has had notable clinical success regarding sustained treatment response for certain types of cancers. Immunotherapy has not been shown to be effective for treatment of pancreatic cancer, primarily due to the existence of a unique tumor microenvironment consisting of an abundant desmoplastic stroma and immunosuppressive immune cells that renders the immune system unable to mount an effective antitumor response. The desmoplastic stroma is thought to act as a physical barrier for effective delivery of cancer therapies, and the tumor microenvironment also contains immunosuppressive cell types and lacks effector $T$ cells that lead to suppressed immune response against the tumor. Pancreatic tumor stroma also contain carcinoma-associated fibroblasts, which express fibroblast activation protein- $\alpha$ (FAP) that further contribute to an immunosuppressive environment [7]. These barriers intrinsic to pancreatic cancer are major contributors to the poor treatment response seen with immunotherapy. Yet, if premalignant mucinous pancreatic cysts lack the tumor microenvironment and immunosuppressive immune cells of pancreatic cancer, they may represent a viable target for immunotherapy. A pilot study evaluating EUS-guided chemoablation with the addition of an immunotherapy agent in patients with premalignant pancreatic cysts would be warranted to validate this approach.

\section{Conclusion}

High rates of complete cyst ablation are noted when pancreatitis is associated with pancreatic cyst ablation. This is evident both in the literature and by the representative case at our institution. Localized activation of the immune system likely plays a role in tumor destruction, and so these lesions may show a robust response to immunotherapy. We propose that further studies are warranted to investigate the utility of adding an immune-activating agent to the chemoablation admixture in an effort to increase rates of complete ablation over that noted currently with chemoablation alone (50\%-79\%). A pilot study involving EUS-guided infusion of an immune-activating agent with one of the currently used chemoablation agents (paclitaxel \pm gemcitabine) for premalignant pancreatic cyst ablation would be warranted to validate this approach.

\section{Competing interests}

Dr. Moyer is a consultant for Boston Scientific.

\section{References}

[1] Moyer MT, Sharzehi S, Mathew A et al. The safety and efficacy of an alcohol-free pancreatic cyst ablation protocol. Gastroenterol 2017; 153: 1295-1303

[2] Dewitt JM, McGreevy K, Schmidt CM et al. EUS-guided ethanol versus saline solution lavage for pancreatic cysts: a randomized, doubleblind study. Gastrointest Endosc 2009; 70: 710-723

[3] Oh HC, Brugge WR. EUS-guided pancreatic cyst ablation: a critical review (with video). Gastrointest Endosc 2013; 77: 526-533

[4] Oh HC, Seo DW, Lee TY et al. New treatment for cystic tumors of the pancreas: EUS guided ethanol lavage with paclitaxel injection. Gastrointest Endosc 2008; 67: 636-642

[5] DeWitt J, DiMaio C], Brugge WR. Long-term follow-up of pancreatic cysts that resolve radiologically after EUS-guided ethanol ablation. Gastrointest Endosc 2010; 72: 862-866

[6] Dewitt JM, Al-Haddad M, Sherman S et al. Alterations in cyst fluid genetics following endoscopic ultrasound-guided pancreatic cyst ablation with ethanol and paclitaxel. Endoscopy 2014; 46: 457-464

[7] Torphy RJ, Zhu Y, Schulick RD. Immunotherapy for pancreatic cancer: Barriers and breakthroughs. Ann Gastroenterol Surg 2018; 2: 274281 